\title{
POTENTIAL NUTRITIVE VALUE OF ASTRAGALUS SPECIES HARVESTED AT THREE DIFFERENT MATURITY STAGES
}

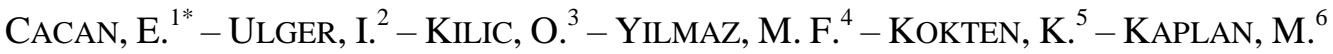 \\ ${ }^{1}$ Department of Crop and Animal Production, Vocational School of Genc, University of Bingol \\ Bingol, Turkey \\ ${ }^{2}$ Department of Animal Science, Faculty of Agriculture, University of Erciyes \\ Kayseri, Turkey \\ ${ }^{3}$ Department of Park and Garden Plants, Vocational School of Technical Sciences, University of \\ Bingol \\ Bingol, Turkey \\ ${ }^{4}$ East Mediterranean Transitional Zone Agricultural Research of Institute \\ Kahramanmaras, Turkey \\ ${ }^{5}$ Department of Field Crops, Faculty of Agriculture, University of Bingol \\ Bingol, Turkey \\ ${ }^{6}$ Department of Field Crops, Faculty of Agriculture, University of Erciyes \\ Kayseri, Turkey \\ *Corresponding author \\ e-mail: erdalcacan@gmail.com; phone: +90-505-844-5280; fax:+90-426-411-2083 \\ (Received 22 ${ }^{\text {nd }}$ Aug 2017; accepted $8^{\text {th }}$ Nov 2017)
}

\begin{abstract}
The present study was conducted to investigate the effects of maturity stages on the chemical composition, in vitro gas and methane production, metabolic energy and organic matter digestibility for eleven Astragalus species widely encountered over the rangelands and intensely grazed by ruminants. Astragalus samples were collected from the rangelands at three different stages namely as before flowering, flowering and bear fruit stages. Dried samples were then subjected to various chemical analysis. Effects of Astragalus species and maturity stages on chemical composition, in vitro gas and methane production, metabolic energy and organic matter digestibility were found to be quite significant $(\mathrm{P}<0.001)$. Acid detergent fiber (ADF) and neutral detergent fiber (NDF) ratios increased, while the crude protein, crude ash, crude oil, condensed tannin contents, gas and methane production levels decreased with the progress of maturity stage. It can be suggested that grazing was more favorable at before flowering or flowering stages of Astragalus species because of high crude protein and metabolic energy content of plant at these stages. Also, although all Astragalus species were considered as a quality feed source for ruminants, endemic Astragalus oocephalus and Astragalus longifolius species were prominent with their superior nutritive properties.
\end{abstract}

Keywords. chemical composition; in vitro gas production; metabolic energy; digestibility; condensed tannin content; grazing

\section{Introduction}

Astragalus with about 2000 species is a member of legumes family and commonly composed of herbaceous and small shrubs. They are used in animal feeds, medicines and pharmaceuticals, in erosion prevention, bee pastures, dye and textile industries (Gruenwald et al., 1998). Astragalus species are widespread in steppes with low precipitation levels and Alpines with low temperatures and commonly grazed by 
ruminants. There is limited information about the nutritional properties and grazing periods of Astragalus species.

Harvest or grazing periods greatly influence nutritive values of feeds. Studies about harvest or grazing periods of these species are of great significant for yield and quality of feed and also for sustainable use of natural resources. Despite these kinds of investigations on several species, there are still a lot of different plant species to be researched (Kamalak and Canbolat, 2010; Kaplan et al., 2014a).

Chemical composition, metabolic energy and digestible nutrients are the significant quality indicators for animal feeds (Canbolat, 2012). For this purpose, in vitro gas production technique developed by Menke et al. (1979) is widely used in recent years to determine nutritional values of feeds under in vitro conditions since the technique is a fast, easy and cheap method (Kaplan et al., 2014b). The gas production technique is also used to determine the methane reduction potential of feeds affecting global warming (Lin et al., 2013). The present study was conducted to investigate the effects of maturity stages on chemical composition, in vitro gas and methane production of different Astragalus species.

\section{Materials and methods}

\section{Plant materials}

Eleven different Astragalus species widespread in Bingol province of Turkey and commonly grazed by ruminants were harvested from the rangelands and natural areas at three different maturity stages (before-flowering, full-flowering, fruit-set). Astragalus gummifer Labill, Astragalus cinereus Willd, Astragalus compactus Lam, Astragalus lineatus Lam. var. longidens, Astragalus oocephalus Boiss subsp. stachyophorus, Astragalus amblolepis, Astragalus declinatus Wild, Astragalus lineatus Lam. var. lineatus, Astragalus longifolius Lam., Astragalus aureus Willd and Astragalus onobrychis species were used as the plant materials of the study. From these species Astragalus oocephalus Boiss subsp. stachyophorus and Astragalus longifolius Lam. are endemic species.

\section{Climate data}

Climate data for Bingol province were received from the nearest meteorological station of Directorate of Meteorology. According the meteorological data of research area, the long-term (2000-2015) monthly average temperature is $12.3{ }^{\circ} \mathrm{C}$, total precipitation is $917.8 \mathrm{~mm}$ and relative humidity is $56.6 \%$. Average temperature, precipitation and relative humidity of the research period (August 2015-August 2016) were quite close to long-term averages $\left(13.9^{\circ} \mathrm{C}, 923.7 \mathrm{~mm}\right.$ and $53.4 \%$, respectively). Soil structure of the Bingol province is clay-loam and loamy (Ates and Turan, 2015).

\section{Chemical analysis}

The Astragalus species were harvested at before flowering, flowering and bear fruit stages. Samples were dried at $70{ }^{\circ} \mathrm{C}$ for $48 \mathrm{~h}$, ground and sieved through $1 \mathrm{~mm}$ sieve. Crude ash content of samples was determined by ashing the samples at $550{ }^{\circ} \mathrm{C}$ for $8 \mathrm{~h}$ in an ash oven. Soxhlet extracting system was used to determine the crude oil content of the samples by ether extraction method (AOAC, 1990). Kjeldahl method was used to determine nitrogen $(\mathrm{N})$ content of the Astragalus species and crude protein level was 
calculated by multiplying N content by 6.25 constant (AOAC, 1990). NDF (Van Soest and Wine, 1967) and ADF (Van Soest, 1963) analysis were performed by using ANKOM 200 Fiber Analyzer (ANKOM Technology Corp. Fairport, NY, USA). Condensed tannin content analysis of the samples was performed by using Butanol-HCl technique (Makkar et al., 1995).

\section{The in vitro total gas and methane production}

In vitro gas production technique developed by Menke et al. (1979) was used to determine in vitro gas and methane production of the samples. The rumen liquid was collected from three fistula installed hogget fed with a mixture $60 \%$ alfalfa and $40 \%$ barley. Liking blocks and clean water was supplied ad-libutum to the hogget. The rumen liquid was collected before feeding in the morning, filtrated through 6-layer cheesecloth to remove solid particles and mixed with twice as much synthetic saliva solution. Approximately $200 \mathrm{mg}$ ground samples were placed in $100 \mathrm{ml}$ glass syringes. The samples were weighed in triplicates. The sample syringes were then supplemented with $30 \mathrm{ml}$ buffered rumen liquid. Three syringes including only buffered rumen liquid as control group and the syringes including samples and buffered rumen liquid were placed into a water bath set at $39{ }^{\circ} \mathrm{C}$. The net gas production was calculated by subtracting gas production of control group from the gas production of sample group. Total gas production was determined as $\mathrm{mL}$ after incubation of Astragalus samples at $39{ }^{\circ} \mathrm{C}$ for $24 \mathrm{~h}$. The methane ratio of the gas was obtained by using an Infrared methane analyzer (Sensor Europe GmbH, Erkrath, Germany) and the following equation (Eq. 1 ) was used to calculate methane production levels (Goel et al., 2008):

$$
\text { Methane production }(\mathrm{mL})=\text { Total gas }(\mathrm{mL}) \times \text { Methane ratio }(\%) \quad \text { (Eq. 1) }
$$

\section{Metabolic energy (ME) and organic matter digestibility (OMD)}

Metabolic energy content of Astragalus samples was calculated in accordance with the following equation (Eq. 2) using some parameters related to $24 \mathrm{~h}$ gas production and chemical composition of samples (Menke and Steingass, 1988). Organic matter digestibility (\%) of the samples was determined by using Eq. 3 as suggested by Menke et al. (1979):

$$
\begin{gathered}
\mathrm{ME}\left(\mathrm{MJ} \mathrm{kg}^{-1} \mathrm{DM}\right)=2.20+0.136 \mathrm{GP}+0.057 \mathrm{CP}+0.002859 \mathrm{CP}^{2} \\
\mathrm{OMD}(\%)=14.88+0.889 \mathrm{GP}+0.45 \mathrm{CP}+0.0651 \mathrm{CA}
\end{gathered}
$$

In these equations; DM: Dry matter, GP: Net gas production after $24 \mathrm{~h}(\mathrm{~mL}), \mathrm{CP}$ : Crude protein ratio (\%), CO: Crude oil ratio (\%), CA: Crude ash ratio (\%) and OMD: Organic matter digestibility (\%).

\section{Statistical analysis}

The experimental design was completely randomized design with 3 replications. Variance and correlation analysis were performed by using Jump SAS (2009) statistical software. Tukey test was used to determine the difference between the means. As a complement of ANOVA procedure, Biplot Analysis was performed using nutritive 
value and gas production parameters as variables and Astragalus species as classification criterion (Yan and Kang, 2003).

\section{Results}

\section{Chemical composition}

The effects of harvest time on nutritional characteristics of different Astragalus species were investigated in this study. Chemical composition of Astragalus samples is provided in Table 1. The effects of Astragalus species, harvest time and Astragalus $\mathrm{x}$ harvest time interaction on chemical composition were found to be highly significant $(\mathrm{P} \leq 0.01)$. ADF and NDF ratios increased and crude protein, crude ash, crude oil and condensed tannin contents decreased with the progress of harvest time. Crude protein ratios varied between 11.65-32.79\%, ADF ratios between 16.22-48.54\%, NDF ratios between $35.62-66.08 \%$, crude oil contents between $0.40-3.43 \%$, crude ash contents between $3.14-11.54 \%$ and condensed tannin contents between $0.32-1.00 \%$.

Table 1. Chemical composition of Astragalus species harvested at different stages

\begin{tabular}{|c|c|c|c|c|c|c|c|}
\hline Astragalus species & Stages & $\mathbf{C P}$ & ADF & NDF & CT & $\mathrm{CO}$ & CA \\
\hline \multirow{4}{*}{$\begin{array}{c}\text { Astragalus gummifer } \\
\text { Labill. }\end{array}$} & Before flowering & $21.7 \mathrm{e}$ & $26.05 \mathrm{~m}$ & $41.14 \mathrm{mn}$ & $0.62 \mathrm{~g}-\mathrm{j}$ & $1.98 \mathrm{~cd}$ & $6.39 \mathrm{i}-1$ \\
\hline & Flowering & $19.42 \mathrm{gh}$ & 28.141 & $42.391 \mathrm{~m}$ & $0.521-\mathrm{o}$ & $1.31 \mathrm{~g}-\mathrm{k}$ & $5.621 \mathrm{mn}$ \\
\hline & Bear fruit & $14.05 \mathrm{r}$ & $32.45 \mathrm{j}$ & $45.01 \mathrm{k}$ & $0.40 \mathrm{pq}$ & $0.921 \mathrm{mn}$ & $5.36 \mathrm{~m}-\mathrm{p}$ \\
\hline & Average & 18.39 I & 28.88 H & $42.85 \mathrm{G}$ & $0.51 \mathrm{G}$ & $1.40 \mathrm{D}$ & $5.79 \mathrm{D}$ \\
\hline \multirow{4}{*}{$\begin{array}{c}\text { Astragalus cinereus } \\
\text { Willd. }\end{array}$} & & $24.82 \mathrm{c}$ & 23.180 & $36.43 p$ & $0.87 \mathrm{~b}$ & $2.36 \mathrm{~b}$ & $8.58 \mathrm{cde}$ \\
\hline & Flowering & $22.41 \mathrm{de}$ & $28.63 \mathrm{kl}$ & 39.32 o & $0.66 f g$ & $1.5 \mathrm{fgh}$ & $7.65 \mathrm{efg}$ \\
\hline & Bear fruit & $19.9 f g$ & $33.69 \mathrm{i}$ & $41.80 \mathrm{~m}$ & $0.49 \mathrm{mno}$ & $0.87 \mathrm{mno}$ & $6.93 \mathrm{~g}-\mathrm{k}$ \\
\hline & Average & $22.37 \mathrm{C}$ & 28.50 I & 39.19 I & $0.68 \mathrm{C}$ & $4.58 \mathrm{C}$ & $7.72 \mathrm{~B}$ \\
\hline \multirow{4}{*}{$\begin{array}{c}\text { Astragalus } \\
\text { compactus Lam }\end{array}$} & & & $42.43 \mathrm{~d}$ & & & & $5.14 n-q$ \\
\hline & Flowering & $18.23 \mathrm{i}-1$ & $46.19 \mathrm{c}$ & $57.3 \mathrm{e}$ & $0.46 o p$ & 0.82 nop & $4.58 \mathrm{o}-\mathrm{r}$ \\
\hline & Bear fruit & $17.261 \mathrm{mn}$ & $47.29 \mathrm{~b}$ & $63.04 \mathrm{~b}$ & $0.39 q$ & $0.59 \mathrm{pq}$ & $3.60 \mathrm{rst}$ \\
\hline & & $19.13 \mathrm{H}$ & $45.30 \mathrm{~A}$ & 57.76 B & $0.47 \mathrm{H}$ & $0.85 G$ & $4.44 \mathrm{~F}$ \\
\hline \multirow{4}{*}{$\begin{array}{l}\text { Astragalus lineatus } \\
\text { Lam. var. longidens }\end{array}$} & Before flowering & $22.11 \mathrm{e}$ & $33.23 \mathrm{ij}$ & $42.17 \mathrm{~m}$ & $0.98 \mathrm{a}$ & $1.71 \mathrm{ef}$ & 7.96def \\
\hline & Flowering & $19.32 \mathrm{ghi}$ & $37.23 \mathrm{~g}$ & $47.95 \mathrm{j}$ & $0.76 \mathrm{cde}$ & $1.14 \mathrm{jkl}$ & $7.23 \mathrm{f}-\mathrm{i}$ \\
\hline & Bear fruit & 17.99jkl & $39.97 \mathrm{e}$ & $51.28 \mathrm{gh}$ & $0.54 \mathrm{k}-\mathrm{n}$ & $0.931 \mathrm{mn}$ & $6.4 \mathrm{i}-1$ \\
\hline & & $19.81 \mathrm{~F}$ & $36.81 \mathrm{D}$ & $47.13 \mathrm{E}$ & $0.76 \mathrm{~B}$ & $1.26 \mathrm{E}$ & $7.20 \mathrm{C}$ \\
\hline \multirow{4}{*}{$\begin{array}{c}\text { Astragalus } \\
\text { oocephalus Boiss } \\
\text { subsp. stachyophorus }\end{array}$} & Before flowering & $32.79 \mathrm{a}$ & $24.63 n$ & $35.62 \mathrm{p}$ & & $1.71 \mathrm{ef}$ & $8.68 \mathrm{~cd}$ \\
\hline & Flowering & $26.64 b$ & $28.91 \mathrm{kl}$ & 39.76 no & $0.56 \mathrm{i}-1$ & $1.40 \mathrm{~g}-\mathrm{j}$ & $6.64 \mathrm{~h}-\mathrm{k}$ \\
\hline & Bear fruit & $22.28 \mathrm{de}$ & $37.99 \mathrm{fg}$ & $49.53 \mathrm{i}$ & $0.32 \mathrm{r}$ & $1.15 \mathrm{jkl}$ & 5.581-o \\
\hline & Average & $27.24 \mathrm{~A}$ & $30.51 \mathrm{~F}$ & $41.64 \mathrm{H}$ & $0.53 \mathrm{G}$ & $1.42 \mathrm{D}$ & $6.96 \mathrm{C}$ \\
\hline \multirow{4}{*}{$\begin{array}{l}\text { Astragalus } \\
\text { amblolepis }\end{array}$} & Before flowering & 16.18nop & $26.94 \mathrm{~m}$ & $58.79 \mathrm{~d}$ & $0.8 \mathrm{~cd}$ & $2.21 b c$ & $4.28 \mathrm{qrs}$ \\
\hline & Flowering & $14.55 \mathrm{qr}$ & $32.87 \mathrm{ij}$ & $62.06 \mathrm{~b}$ & $0.56 \mathrm{i}-\mathrm{m}$ & $1.7 \mathrm{ef}$ & $3.14 \mathrm{t}$ \\
\hline & Bear fruit & $11.65 \mathrm{~s}$ & $35.86 h$ & $66.08 \mathrm{a}$ & $0.37 \mathrm{qr}$ & $1.23 \mathrm{i}-\mathrm{k}$ & $3.36 \mathrm{st}$ \\
\hline & Average & $14.13 \mathrm{~K}$ & 31.89 E & $62.31 \mathrm{~A}$ & $0.58 \mathrm{E}$ & $1.71 \mathrm{~B}$ & $3.60 \mathrm{~F}$ \\
\hline
\end{tabular}




\begin{tabular}{|c|c|c|c|c|c|c|c|}
\hline \multirow{4}{*}{$\begin{array}{c}\text { Astragalus declinatus } \\
\text { Wild }\end{array}$} & Before flowering & $22.53 \mathrm{de}$ & $27.08 \mathrm{~m}$ & $36.2 p$ & $0.67 f g$ & $3.43 \mathrm{a}$ & $11.54 \mathrm{a}$ \\
\hline & Flowering & $20.57 f$ & $29.44 \mathrm{k}$ & 40.37 no & $0.57 \mathrm{i}-1$ & $1.48 \mathrm{f}-\mathrm{i}$ & $10.15 b$ \\
\hline & Bear fruit & $17.41 \mathrm{klm}$ & $33.46 \mathrm{ij}$ & $47.81 \mathrm{j}$ & $0.521-\mathrm{o}$ & $0.901 \mathrm{mn}$ & $9.14 \mathrm{c}$ \\
\hline & Average & 20.17 E & $30.00 \mathrm{G}$ & $41.46 \mathrm{H}$ & $0.58 \mathrm{E}$ & $1.94 \mathrm{~A}$ & $10.28 \mathrm{~A}$ \\
\hline \multirow{4}{*}{$\begin{array}{l}\text { Astragalus lineatus } \\
\text { Lam. var. lineatus }\end{array}$} & Before flowering & $22.55 \mathrm{de}$ & $35.73 \mathrm{~h}$ & $45.72 \mathrm{k}$ & $0.76 \mathrm{cde}$ & $2.21 b c$ & $7.34 \mathrm{f}-\mathrm{i}$ \\
\hline & Flowering & $20.24 f g$ & $43.06 \mathrm{~d}$ & $50.37 \mathrm{hi}$ & $0.65 \mathrm{fgh}$ & $1.22 \mathrm{ijk}$ & $7.14 f-j$ \\
\hline & Bear fruit & $18.5 \mathrm{~h}-\mathrm{k}$ & $45.6 \mathrm{c}$ & $52.17 \mathrm{fg}$ & $0.521-\mathrm{o}$ & 0.83 nop & $6.58 \mathrm{~h}-1$ \\
\hline & Average & 20.43 D & $41.46 \mathrm{~B}$ & 49.42 D & $0.65 \mathrm{D}$ & $1.42 \mathrm{D}$ & $7.02 \mathrm{C}$ \\
\hline \multirow{4}{*}{$\begin{array}{c}\text { Astragalus } \\
\text { longifolius Lam. }\end{array}$} & Before flowering & $26.43 b$ & $28.74 \mathrm{kl}$ & $36.71 p$ & $1.00 \mathrm{a}$ & $1.82 \mathrm{de}$ & $7.7 \mathrm{~d}-\mathrm{g}$ \\
\hline & Flowering & $23.27 \mathrm{~d}$ & $35.84 \mathrm{~h}$ & 43.601 & $0.75 \mathrm{de}$ & 0.74 nop & $6.98 \mathrm{f}-\mathrm{k}$ \\
\hline & Bear fruit & $19.3 \mathrm{ghi}$ & $48.54 \mathrm{a}$ & $52.17 \mathrm{fg}$ & $0.59 \mathrm{~h}-\mathrm{k}$ & $0.59 \mathrm{pq}$ & $6.04 \mathrm{k}-\mathrm{n}$ \\
\hline & Average & 23.00 B & $37.70 \mathrm{C}$ & $44.16 \mathrm{~F}$ & $0.78 \mathrm{~A}$ & $1.05 \mathrm{~F}$ & $6.91 \mathrm{C}$ \\
\hline \multirow{4}{*}{$\begin{array}{l}\text { Astragalus aureus } \\
\text { Willd. }\end{array}$} & Before flowering & 18.61hij & $24.56 n$ & $52.00 \mathrm{fg}$ & $0.83 b c$ & $1.52 \mathrm{fg}$ & $5.12 n-q$ \\
\hline & Flowering & $16.62 \mathrm{mno}$ & $28.42 \mathrm{kl}$ & $57.28 \mathrm{e}$ & 0.47 no & $1.11 \mathrm{klm}$ & $4.52 \mathrm{pqr}$ \\
\hline & Bear fruit & $15.34 \mathrm{pq}$ & $38.42 \mathrm{f}$ & $60.64 c$ & $0.35 \mathrm{qr}$ & $0.40 q$ & $4.14 \mathrm{q}-\mathrm{t}$ \\
\hline & Average & $16.85 \mathrm{~J}$ & $30.47 \mathrm{~F}$ & 56.64 C & 0.55 F & $1.01 \mathrm{~F}$ & $4.59 \mathrm{E}$ \\
\hline \multirow{4}{*}{$\begin{array}{l}\text { Astragalus } \\
\text { onobrychis }\end{array}$} & Before flowering & 22.56de & $16.22 \mathrm{r}$ & $45.32 \mathrm{k}$ & $0.75 \mathrm{de}$ & $1.25 \mathrm{~h}-\mathrm{k}$ & $7.45 \mathrm{fgh}$ \\
\hline & Flowering & $20.07 f g$ & $18.31 \mathrm{q}$ & $47.14 \mathrm{j}$ & $0.63 \mathrm{ghi}$ & $0.63 \mathrm{opq}$ & $7.05 f-j$ \\
\hline & Bear fruit & 15.97op & $20.53 p$ & $49.58 \mathrm{i}$ & $0.58 \mathrm{~h}-1$ & $0.43 q$ & $6.17 \mathrm{j}-\mathrm{m}$ \\
\hline & Average & 19.54 G & $18.35 \mathrm{~J}$ & 47.32 E & $0.65 \mathrm{D}$ & 0.77 H & $6.89 \mathrm{C}$ \\
\hline
\end{tabular}

CP: crude protein (\%); ADF: acid detergent fiber (\%); NDF: Neutral detergent fiber (\%); CT: condense tannin (\%); CO: crude oil (\%); CA: crude ash (\%);small letters show significant differences between the interactions of Astragalus species and harvest stage; capital letters show significant differences between the means of Astragalus species

\section{Gas and methane production, metabolic energy and organic matter digestibility}

Average gas production, methane production, metabolic energy and organic matter digestibility of Astragalus species harvested at different vegetation stages are provided in Table 2. The effects of Astragalus species, harvest times and Astragalus x harvest time interaction on gas and methane production, ME and OMD levels were found to be highly significant $(\mathrm{P} \leq 0.01)$. Gas and methane production, $\mathrm{ME}$ and $\mathrm{OMD}$ decreased with the progress of harvest time. Gas production values varied between 31.50-50.00 $\mathrm{ml}$, methane productions between 3.31-6.54 ml, metabolic energy contents between 7.19-10.43 $\mathrm{MJ} \mathrm{kg}^{-1} \mathrm{DM}$ and organic matter digestibility levels between 50.47-69.52\%.

Table 2. Gas and methane production, metabolic energy and organic matter digestibility of Astragalus species harvested at different stages

\begin{tabular}{c|c|c|c|c|c}
\hline Astragalus species & Stages & GP & CH $_{\mathbf{4}}$ & ME & OMD \\
\hline \multirow{4}{*}{ Astragalus gummifer } & Before flowering & $46.50 \mathrm{cde}$ & $6.00 \mathrm{~b}$ & $9.98 \mathrm{~d}$ & $66.40 \mathrm{bc}$ \\
Labill. & Flowering & $39.00 \mathrm{jk}$ & $4.69 \mathrm{f}$ & $8.64 \mathrm{j}$ & $58.65 \mathrm{j}$ \\
& Bear fruit & $34.00 \mathrm{op}$ & $3.78 \mathrm{i}$ & $7.48 \mathrm{op}$ & $51.78 \mathrm{qr}$ \\
& Average & $\mathbf{3 9 . 8 3} \mathbf{D}$ & $\mathbf{4 . 8 2} \mathbf{E F}$ & $\mathbf{8 . 7 0} \mathbf{~ E F}$ & $\mathbf{5 8 . 9 4} \mathbf{~ E F}$ \\
\hline
\end{tabular}




\begin{tabular}{|c|c|c|c|c|c|}
\hline $\begin{array}{c}\text { Astragalus cinereus } \\
\text { Willd. }\end{array}$ & $\begin{array}{c}\text { Before flowering } \\
\text { Flowering } \\
\text { Bear fruit } \\
\text { Average }\end{array}$ & $\begin{array}{c}41.50 \mathrm{hi} \\
37.00 \mathrm{k}-\mathrm{n} \\
33.00 \mathrm{pq} \\
\mathbf{3 7 . 1 7} \mathbf{~ F}\end{array}$ & $\begin{array}{l}5.41 \mathrm{~cd} \\
4.28 \mathrm{~g} \\
3.64 \mathrm{I} \\
\mathbf{4 . 4 4 ~ G}\end{array}$ & $\begin{array}{l}9.58 \mathrm{e} \\
8.62 \mathrm{jk} \\
7.76 \mathrm{no} \\
\mathbf{8 . 6 5} \mathbf{F}\end{array}$ & $\begin{array}{c}63.50 \mathrm{~d}-\mathrm{g} \\
58.35 \mathrm{jk} \\
53.62 \mathrm{opq} \\
\mathbf{5 8 . 4 9} \mathbf{~ F}\end{array}$ \\
\hline $\begin{array}{c}\text { Astragalus compactus } \\
\text { Lam }\end{array}$ & $\begin{array}{c}\text { Before flowering } \\
\text { Flowering } \\
\text { Bear fruit } \\
\text { Average }\end{array}$ & $\begin{array}{c}50.00 \mathrm{a} \\
45.50 \mathrm{def} \\
38.00 \mathrm{jkl} \\
\mathbf{4 4 . 5 0 \mathrm { AB }}\end{array}$ & $\begin{array}{c}6.22 \mathrm{ab} \\
5.30 \mathrm{~cd} \\
3.76 \mathrm{I} \\
\mathbf{5 . 1 2} \mathbf{B C}\end{array}$ & $\begin{array}{l}10.32 \mathrm{ab} \\
9.37 \mathrm{efg} \\
8.22 \mathrm{~lm} \\
\mathbf{9 . 3 0} \mathbf{B C}\end{array}$ & $\begin{array}{c}69.52 \mathrm{a} \\
63.83 \mathrm{def} \\
56.67 \mathrm{klm} \\
\mathbf{6 3 . 3 4} \mathbf{B}\end{array}$ \\
\hline $\begin{array}{l}\text { Astragalus lineatus } \\
\text { Lam. var. longidens }\end{array}$ & $\begin{array}{c}\text { Before flowering } \\
\text { Flowering } \\
\text { Bear fruit } \\
\text { Average }\end{array}$ & $\begin{array}{c}49.50 \mathrm{ab} \\
44.00 \mathrm{fg} \\
37.00 \mathrm{k}-\mathrm{n} \\
\mathbf{4 3 . 5 0} \mathbf{B C} \\
\end{array}$ & $\begin{array}{c}6.35 \mathrm{a} \\
5.11 \mathrm{de} \\
4.26 \mathrm{~g} \\
\mathbf{5 . 2 4} \mathbf{A B} \\
\end{array}$ & $\begin{array}{r}10.37 \mathrm{a} \\
9.30 \mathrm{e}-\mathrm{h} \\
8.19 \mathrm{~lm} \\
9.29 \mathrm{BC} \\
\end{array}$ & $\begin{array}{c}69.35 \mathrm{a} \\
63.16 \mathrm{e}-\mathrm{h} \\
56.29 \mathrm{lmn} \\
\mathbf{6 2 . 9 3} \mathbf{B} \\
\end{array}$ \\
\hline $\begin{array}{c}\text { Astragalus } \\
\text { oocephalus Boiss } \\
\text { subsp. stachyophorus }\end{array}$ & $\begin{array}{c}\text { Before flowering } \\
\text { Flowering } \\
\text { Bear fruit } \\
\text { Average }\end{array}$ & $\begin{array}{c}41.50 \mathrm{hi} \\
39.00 \mathrm{jk} \\
35.50 \mathrm{mno} \\
\mathbf{3 8 . 6 7} \mathbf{E} \\
\end{array}$ & $\begin{array}{c}5.24 \mathrm{~cd} \\
4.72 \mathrm{f} \\
3.68 \mathrm{I} \\
\mathbf{4 . 5 5} \mathbf{G} \\
\end{array}$ & $\begin{array}{c}10.05 \mathrm{bcd} \\
9.19 \mathrm{f}-\mathrm{I} \\
8.34 \mathrm{kl} \\
\mathbf{9 . 1 9} \mathrm{C} \\
\end{array}$ & $\begin{array}{c}67.09 \mathrm{bc} \\
61.97 \mathrm{f}-\mathrm{i} \\
56.83 \mathrm{j}-\mathrm{m} \\
\mathbf{6 1 . 9 7} \mathbf{C} \\
\end{array}$ \\
\hline Astragalus amblolepis & $\begin{array}{c}\text { Before flowering } \\
\text { Flowering } \\
\text { Bear fruit } \\
\text { Average }\end{array}$ & $\begin{array}{c}43.00 \mathrm{gh} \\
39.50 \mathrm{ij} \\
35.00 \mathrm{nop} \\
\mathbf{3 9 . 1 7} \mathrm{DE}\end{array}$ & $\begin{array}{l}5.37 \mathrm{~cd} \\
4.83 \mathrm{ef} \\
3.90 \mathrm{hi} \\
\mathbf{4 . 7 0} \mathbf{~ F} \\
\end{array}$ & $\begin{array}{l}9.12 \text { ghi } \\
8.42 \mathrm{jkl} \\
7.50 \mathrm{o} \\
\mathbf{8 . 3 5} \mathbf{~ G} \\
\end{array}$ & $\begin{array}{c}60.66 \mathrm{i} \\
56.75 \mathrm{klm} \\
51.46 \mathrm{r} \\
\mathbf{5 6 . 2 9} \mathbf{G} \\
\end{array}$ \\
\hline $\begin{array}{c}\text { Astragalus declinatus } \\
\text { Wild }\end{array}$ & $\begin{array}{c}\text { Before flowering } \\
\text { Flowering } \\
\text { Bear fruit } \\
\text { Average }\end{array}$ & $\begin{array}{l}45.50 \mathrm{def} \\
44.00 \mathrm{fg} \\
39.00 \mathrm{jk} \\
\mathbf{4 2 . 8 3 ~ C}\end{array}$ & $\begin{array}{l}5.51 \mathrm{c} \\
5.15 \mathrm{de} \\
4.13 \mathrm{gh} \\
4.93 \mathrm{CD}\end{array}$ & $\begin{array}{c}10.15 \mathrm{a}-\mathrm{d} \\
9.45 \mathrm{ef} \\
8.42 \mathrm{jkl} \\
\mathbf{9 . 3 4} \mathbf{B}\end{array}$ & $\begin{array}{c}66.22 \mathrm{c} \\
63.91 \mathrm{de} \\
57.98 \mathrm{jkl} \\
\mathbf{6 2 . 7 0} \mathbf{B C}\end{array}$ \\
\hline $\begin{array}{l}\text { Astragalus lineatus } \\
\text { Lam. var. lineatus }\end{array}$ & $\begin{array}{c}\text { Before flowering } \\
\text { Flowering } \\
\text { Bear fruit } \\
\text { Average }\end{array}$ & $\begin{array}{l}48.00 \mathrm{abc} \\
37.50 \mathrm{j}-\mathrm{m} \\
33.50 \mathrm{opq} \\
\text { 39.67 DE }\end{array}$ & $\begin{array}{l}6.38 \mathrm{a} \\
4.65 \mathrm{f} \\
3.86 \mathrm{hi} \\
4.96 \mathrm{D}\end{array}$ & $\begin{array}{c}10.29 \mathrm{abc} \\
8.48 \mathrm{jkl} \\
7.72 \mathrm{no} \\
\mathbf{8 . 8 3} \mathbf{D E}\end{array}$ & $\begin{array}{l}68.18 \mathrm{ab} \\
57.79 \mathrm{jkl} \\
53.10 \mathrm{pq} \\
\mathbf{5 9 . 8 0} \mathbf{E}\end{array}$ \\
\hline $\begin{array}{l}\text { Astragalus longifolius } \\
\text { Lam. }\end{array}$ & $\begin{array}{c}\text { Before flowering } \\
\text { Flowering } \\
\text { Bear fruit } \\
\text { Average }\end{array}$ & $\begin{array}{l}47.50 \mathrm{bcd} \\
44.50 \mathrm{efg} \\
42.50 \mathrm{gh} \\
\mathbf{4 4 . 8 3 \mathrm { A }} \\
\end{array}$ & $\begin{array}{c}5.96 \mathrm{~b} \\
4.69 \mathrm{f} \\
4.32 \mathrm{~g} \\
4.99 \mathrm{DC}\end{array}$ & $\begin{array}{l}10.43 \mathrm{a} \\
9.59 \mathrm{e} \\
8.99 \mathrm{I} \\
\mathbf{9 . 6 7} \mathrm{A} \\
\end{array}$ & $\begin{array}{c}69.50 \mathrm{q} \\
65.37 \mathrm{~cd} \\
61.74 \mathrm{ghi} \\
\mathbf{6 5 . 5 4 \mathbf { A }} \\
\end{array}$ \\
\hline $\begin{array}{l}\text { Astragalus aureus } \\
\text { Willd. }\end{array}$ & $\begin{array}{c}\text { Before flowering } \\
\text { Flowering } \\
\text { Bear fruit } \\
\text { Average }\end{array}$ & $\begin{array}{c}49.00 \mathrm{ab} \\
43.50 \mathrm{fgh} \\
36.50 \mathrm{lmn} \\
\mathbf{4 3 . 0 0} \mathbf{C}\end{array}$ & $\begin{array}{l}6.54 \mathrm{a} \\
5.49 \mathrm{c} \\
3.74 \mathrm{I} \\
\mathbf{5 . 2 6 ~ A}\end{array}$ & $\begin{array}{l}10.01 \mathrm{~cd} \\
9.03 \mathrm{hi} \\
7.83 \mathrm{n} \\
\mathbf{8 . 9 6} \mathbf{D}\end{array}$ & $\begin{array}{c}67.15 \mathrm{bc} \\
61.32 \mathrm{hi} \\
54.50 \mathrm{nop} \\
60.99 \mathrm{D}\end{array}$ \\
\hline $\begin{array}{l}\text { Astragalus } \\
\text { onobrychis }\end{array}$ & $\begin{array}{c}\text { Before flowering } \\
\text { Flowering } \\
\text { Bear fruit } \\
\text { Average }\end{array}$ & $\begin{array}{c}41.50 \mathrm{hi} \\
35.00 \mathrm{nop} \\
31.50 \mathrm{q} \\
\mathbf{3 6 . 0 0 ~ G}\end{array}$ & $\begin{array}{l}5.13 \mathrm{de} \\
4.23 \mathrm{~g} \\
3.31 \mathrm{j} \\
\mathbf{4 . 2 2} \mathbf{H}\end{array}$ & $\begin{array}{c}9.21 \mathrm{f}-\mathrm{i} \\
8.01 \mathrm{mn} \\
7.19 \mathrm{p} \\
\mathbf{8 . 1 4} \mathbf{H}\end{array}$ & $\begin{array}{c}62.41 \mathrm{e}-\mathrm{i} \\
55.48 \mathrm{mno} \\
50.47 \mathrm{r} \\
\mathbf{5 6 . 1 2} \mathbf{~ G}\end{array}$ \\
\hline
\end{tabular}

GP: gas production (ml); $\mathbf{C H}_{4}$ : methane (ml); ME: metabolic energy (MJ kg-1 DM); OMD: organic matter digestibility (\%); small letters show significant differences between the interactions of Astragalus species and harvest stage; capital letters show significant differences between the means of Astragalus species 


\section{Biplot analysis}

Biplot analysis revealed that two principle components were able to explain $73.10 \%$ of total variation ( $41.80 \%$ by PC1 and $31.30 \%$ by PC2). As can be seen in Figure 1; crude ash, crude protein and condensed tannin parameters constituted one group; ME and OMD constituted one group and GP, methane and ADF constituted another group. Crude oil and NDF alone separately constituted different groups. Astragalus longifolius was prominent with ME and OMD, Astragalus amblolepis with NDF, Astragalus oocephalus with crude protein, Astragalus compactus with ADF and Astragalus aureus was prominent with methane production (Table1, Table 2, Figure 1).

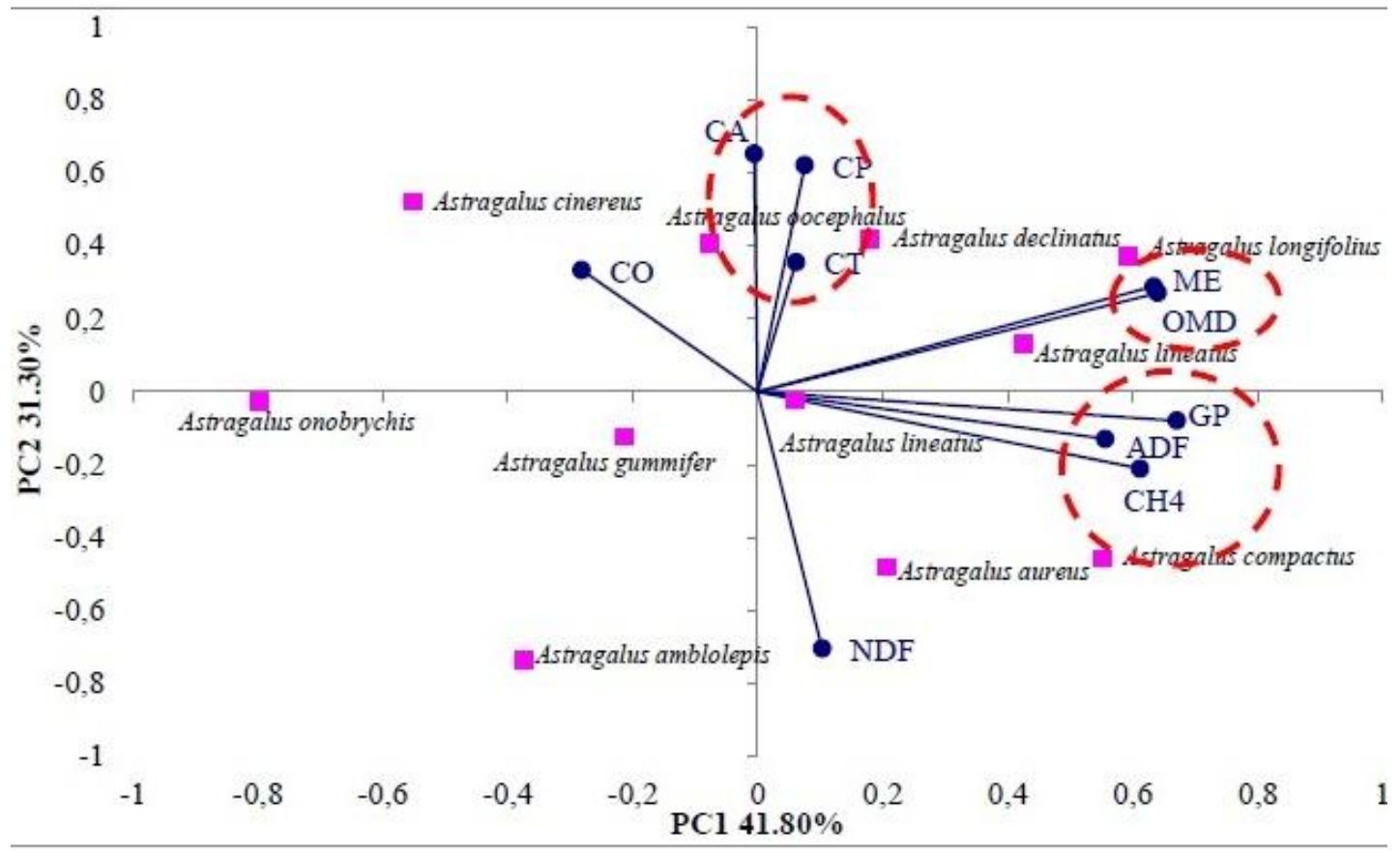

Figure 1. The biplot for nutritive values of Astragalus species

\section{Discussion}

Crude protein content is a significant indicator of feed quality (Assefa and Ledin, 2001). It was reported that differences in crude protein contents of different varieties can be resulted from genetics of the plants and such values can also vary depending on leaf, spike and stem ratios, ripening periods, fertilization, climate and soil conditions (Ball et al., 2011). Number of leaves and thus leaf/stalk ratios decrease with the progress of ripening. Ripening reduces number of leaves rich in crude protein and reduces crude protein ratios of the plants (Buxton, 1996). In present study, crude protein ratios of Astragalus species decrease with the progress of ripening and quite significant differences were observed among the species. The crude protein contents of the present study ranged within the values reported by Davis (1982) for 33 different Astragalus species. However, crude protein content (32.79\%) of endemic Astragalus oocephalus at before flowering period was higher than those reported values.

The differences in stem and leaf ratios in plants result in differences also in crude protein contents, ADF and NDF ratios. ADF and NDF levels, which are the cell wall components, increased with the progress of ripening. It was reported for several plant 
species that increased ADF and NDF ratios reduced crude protein, crude oil and carbohydrate levels of the plants with the progress of vegetation period (Canbolat, 2012; Kaplan et al., 2014a, 2014b).

It was reported that low condensed tannin levels (2-3\%) had beneficial effects because they prevent extreme decomposition of proteins in rumen (Barry, 1987). Kumar and Singh (1984) reported that high condensed tannin levels had harmful effects because they decrease protein digestibility. In the current study, condensed tannin levels $(0.32-1.00 \%)$ were lower than those specified ones and thus such levels had beneficial effects. Crude ash is the residual unburnt portion after ashing dry matter in an as oven (Genctan, 1998). Since it cannot be synthesized by animal organisms, they should be taken externally.

Gas production of Astragalus species were different because of the differences in crude protein, crude oil, ADF and NDF ratios resulted from differences in their stem and leaf ratios. The increased crude oil and crude protein levels caused an increase in the metabolic energy. In vitro gas productions through fermentation in feeds are directly related to the fermentable carbohydrate content. The differences in ME and OMD levels of Astragalus species were mainly resulted from differences in their gas production, crude protein, crude ash and crude oil contents as well as harvest period-induced reductions in these parameters. Decreased fermentable carbohydrate contents with the progress of ripening and differences in stem and leaf ratios also decreased in vitro gas production levels (Blummel and Orskov, 1993). The gas production and thus carbohydrate levels decrease with the progress of ripening.

The feeds are classified according to the methane content after fermentation as low anti-methanogenic $(>11 \%$ and $\leq 14 \%)$, medium anti-methanogenic $(>6 \%$ and $<11 \%)$ and high anti-methanogenic $(>0 \%$ and <6\%) (Lopez et al., 2010). Accordingly, Astragalus species of the present study were classified as high anti-methanogenic.

\section{Conclusion}

It was concluded based on present findings that nutritive values of Astragalus species were quite different from each other and the progress of growing stages affected the nutritive profile of Astragalus samples significantly. Just because of high protein and ME contents, it was recommended that Astragalus species should be grazed in before flowering or full flowering stages. Among 11 Astragalus species investigated in this study, the endemic Astragalus oocephalus and Astragalus longifolius were found to be prominent with their high crude protein content and ME levels and low ADF and NDF ratios. It was observed that Astragalus species had superior properties for the animals. Further research is recommended on nutritive values of different Astragalus species with new in vivo and in vitro studies and also on the effects of Astragalus species on animal health.

\section{REFERENCES}

[1] AOAC (1990): Official Method of Analysis, 15th ed. - Association of Official Analytical Chemists, Washington, DC.

[2] Assefa, G., Ledin, I. (2001): Effect of variety, soil type and fertilizer on the establishment, growth, forage yield, quality and voluntary intake by cattle of oats and 
vetches cultivated in pure stand and mixtures. - Animal Feed Science and Technology 92(1-2): 95-111. DOI: 10.1016/S0377-8401(01)00242-5.

[3] Ates, K., Turan, V. (2015): Some soil characteristics and the fertility status of agricultural soils in Bingol central district. - Turk J Agric Res 2(2): 108-113.

[4] Ball, D. M., Collins, M., Lacefield, G. D., Martin, N. P., Mertens, D. A., Olson, K. E., Putnam, D. H., Undersander, D. J., Wolf, M. W. (2011): Understanding Forage Quality. American Farm Bureau Federation Publication 1-01, Park Ridge, IL.

[5] Barry, T. N. (1987): Secondary Compounds of Forages. - In: Hacker, J. B., Ternouth, J. H. (eds.) Nutrition of Herbivores. Academic Press, Sydney.

[6] Blummel, M., Orskov, E. R. (1993): Comparison of an in vitro gas production and nylon bag degradability of roughages in predicting feed in take in cattle. - Animal Feed Science and Technology 40(2-3): 109-119. DOI: 10.1016/0377-8401(93)90150-I.

[7] Buxton, D. R. (1996): Quality related characteristics of forages as influenced by plant environment and agronomic factors. - Animal Feed Science and Technology 59(1-3): 3749. DOI: 10.1016/0377-8401(95)00885-3.

[8] Canbolat, O. (2012): Comparison of in vitro gas production, organic matter digestibility, relative feed value and metabolizable energy contents of some cereal forages. - Kafkas Univ Vet Fak Derg 18(4): 571-577. DOI:10.9775/kvfd.2011.5833.

[9] Davis, A. M. (1982): Crude protein, crude fiber, tannin and oxalate concentrations of 33 Astragalus species. - Journal of Range Management 35(1): 32-34. DOI: 10.2134/agronj1973.00021962006500040025x.

[10] Genctan, T. (1998): Agricultural Ecology. - Field Crops Department, Tekirdag Agriculture Faculty, Trakya University, Tekirdag.

[11] Goel, G., Makkar, H. P. S., Becker, K. (2008): Effect of Sesbania sesban and Carduus pycnocephalus leaves and fenugreek (Trigonella foenum-graecum L.) seeds and their extraction partitioning of nutrients from roughage-and concentrate-based feeds to methane. - Animal Feed Science and Technology 147(1-3): 72-89. DOI: 10.1016/j.anifeedsci.2007.09.010.

[12] Gruenwald, J., Brendler, T., Jaenicke, C. (1998): PDR for Herbal Medicines. - Medical Economics Company, Montvale, NJ.

[13] Jump, SAS (2009): JMP Statistics and Graphics Guide, Version 8.0 (trial). - SAS Institute Inc, Cary, NC.

[14] Kamalak, A., Canbolat, O. (2010): Determination of nutritive value of wild narrowleaved clover (Trifolium angustifolium) hay harvested at three maturity stages using chemical composition and in vitro gas production. - Tropical Grasslands 44(2):128-133.

[15] Kaplan, M., Kamalak, A., Ozkan, C. O., Atalay, A. I. (2014a): Effect of vegetative stages on potential nutritive value, methane production and condensed tannin content of Onobrychis caput-galli hay. - Harran Univ Vet Fak Derg 3(1): 1-5.

[16] Kaplan, M., Kamalak, A., Kasra, A. A., Guven, I. (2014b): Effect of maturity stages on potential nutritive value, methane production and condensed tannin content of Sanguisorba minor hay. - Kafkas Univ Vet Fak Derg 20(3): 445-449. DOI: $10.9775 / \mathrm{kvfd} .2013 .10383$.

[17] Kumar, R., Singh, M. (1984): Tannins: their adverse role in ruminant nutrition. - J Agric Food Chem 32(3): 447-453. DOI: 10.1021/jf00123a006.

[18] Lin, B., Wang, J. H., Lu, Y., Liang, Q., Liu, J. X. (2013): In vitro rumen fermentation and methane production are influenced by active components of essential oils combined with fumarate. - Journal of Animal Physiology and Animal Nutrition 97(1): 1-9. DOI: 10.1111/j.1439-0396.2011.01236.x.

[19] Lopez, S., Makkar, H. P. S., Soliva, C. R. (2010): Screening Plants and Plant Products for Methane Inhibitors. - In: Vercoe, P. E., Makkar, H. P. S., Schlink, A. (eds.) In Vitro Screening of Plant Resources for Extra-nutritional Attributes in Ruminants: Nuclear and Related Methodologies. Springer, London, New York. 
[20] Makkar, H. P. S., Blummel, M., Becker, K. (1995): Formation of complexes between polyvinyl pyrrolidones or polyethylene glycols and their implication in gas production and true digestibility in vitro techniques. - British Journal of Nutrition 73(6): 897-913. DOI: 10.1079/BJN19950095.

[21] Menke, K. H., Steingass, H. (1988): Estimation of the energetic feed value obtained from chemical analysis and in vitro gas production using rumen fluid. - Animal Research and Development 28: 7-55.

[22] Menke, K. H., Raab, L., Salewski, A., Steingass, H., Fritz, D., Schneider, W. (1979): The estimation of the digestibility and metabolisable energy content of ruminant feeding stuffs from the gas production when they are incubated with rumen liquor in vitro. - The Journal of Agricultural Science 93(1): 217-222. DOI:10.1017/S0021859600086305.

[23] Van Soest, P. J. (1963): The use of detergents in the analysis of fibre feeds. II. A rapid method for the determination of fiber and lignin. - Journal of the A.O.A.C. 46(5): 829835.

[24] Van Soest, P. J., Wine, R. H. (1967): The use of detergents in the analysis of fibrous feeds. IV. Determination of plant cell wall constituents. - Journal of the A.O.A.C. 50(1): 50-55.

[25] Yan, W., Kang, M. S. (2003): GGE-Biplot Analysis: A Graphical Tool for Breeders, Geneticists and Agronomists. - CRD Press, Boca Raton. 